\title{
A Mini Review on Microcystins and Bacterial Degradation
}

\author{
Isaac Yaw Massey and Fei Yang *(D) \\ Department of Occupational and Environmental Health, Xiangya School of Public Health, Central South \\ University, Changsha 410078, China; mriymassey@csu.edu.cn \\ * Correspondence: phfyang@csu.edu.cn
}

Received: 26 March 2020; Accepted: 11 April 2020; Published: 21 April 2020

\begin{abstract}
Microcystins (MCs) classified as hepatotoxic and carcinogenic are the most commonly reported cyanobacterial toxins found in the environment. Microcystis sp. possessing a series of MC synthesis genes (mcyA-mcyJ) are well documented for their excessive abundance, numerous bloom occurrences and MC producing capacity. About 246 variants of MC which exert severe animal and human health hazards through the inhibition of protein phosphatases (PP1 and PP2A) have been characterized. To minimize and prevent MC health consequences, the World Health Organization proposed $1 \mu \mathrm{g} / \mathrm{L} \mathrm{MC}$ guidelines for safe drinking water quality. Further the utilization of bacteria that represent a promising biological treatment approach to degrade and remove MC from water bodies without harming the environment has gained global attention. Thus the present review described toxic effects and bacterial degradation of MCs.
\end{abstract}

Keywords: microcystins; toxicity and carcinogenicity; bacterial degradation; degrading mechanism

Key Contribution: The review highlights toxicity and carcinogenicity of microcystins and will further expand reader's knowledge on bacterial degradation of these toxins.

\section{Introduction}

Cyanobacteria are organisms that inhabit surface and bottom water. These organisms can accumulate to form blooms and scums which are mostly found on the water surface. WHO [1] reported that blooms of toxic cyanobacteria are gradually increasing worldwide in both frequency and severity. While cyanobacterial blooms occur naturally in water bodies, the combination of environmental factors such as nutrients (nitrogen and phosphorus), weather conditions, carbon dioxide, water body, salinity, sunlight, $\mathrm{pH}$, heavy metals, brief periods of drought and heavy rain may trigger for the proliferation of the blooms [2-6]. Cyanobacterial blooms have become a serious global environmental problem in both developing and developed countries due to the unpleasant odor, taste and cyanobacterial toxins produced. The presence of these toxins may reduce water quality, accumulate and magnify in food chains, and bring about significant negative effects on human health and animals [7-10]. Of the numerous cyanobacterial toxins discovered, microcystins (MCs) are classified as hepatotoxic and potentially carcinogenic [11], most often present in water [12] and extensively studied in terms of degradation and removal [13-17]. Thus this review focuses on toxic effects and bacterial degradation of MCs. 


\section{Microcystins}

\subsection{Microcystins Synthesis}

Microcystins are cyclic heptapeptide hepatotoxins primarily found in marine and freshwaters worldwide [8,9,18,19]. Metcalf et al. [20] also indicated that MCs can be produced in desert environments. More than 30 cyanobacterial species are capable to produce MCs [21]. Interestingly much attention has been given to Microcystis sp. owing to its extreme abundance, frequent bloom occurrences and ability to generate MCs [18,22-25]. Microcystis sp. possess a series of MC synthesis genes (mcyA-mcyJ), where mcyA, mcyB and mcyC genes are usually used in detecting toxigenic cyanobacteria $[18,26,27]$. Thus the ability for cyanobacteria to produce MC is determined by the mcy cluster. During the growth, MCs are retained in cyanobacterial cells and are found to be released during senescence and breakdown processes [28-30].

\subsection{Chemical Properties}

Approximately 246 variants of MC have been characterized, which exhibit different degrees of toxicity [31]. Of the numerous MC variants characterized, MC-LR, MC-RR and MC-YR are the most frequently found in the environment, very toxic and extensively studied. MC-LF and MC-LW have also been shown with quite high concentrations [14,18,32-34]. In general MCs share a common cyclic structure cyclo-(-D-Ala-L-X-D-MeAsp-L-Z-Adda-D-Glu-Mdha) (Figure 1) which makes them capable to resist physical and chemical factors. $X$ and $Z$ represent highly variable amino acids, D-MeAsp represents D-erythro-b-methylaspartic acid, Adda represents (2S, 3S, 8S, 9S) 3- amino-9 methoxy-2,6,8-trimethyl-10-phenyldeca-4, 6-dienoic acid and Mdha represents $\mathrm{N}$-methyldehydroalanine. The unique structure Adda is crucial for biological activity of MC molecules [8]. Further the variants of MC mainly differ in X and Z amino acids, and methylation or demethylation on MeAsp and Mdha. For instance MC-LR is known to contain Leucine (L) and Arginine ( $R$ ) amino acids, MC-RR consists of two ' $R$ ' amino acids and MC-YR contains Tyrosine (Y) and ' $R$ ' amino acids [8].

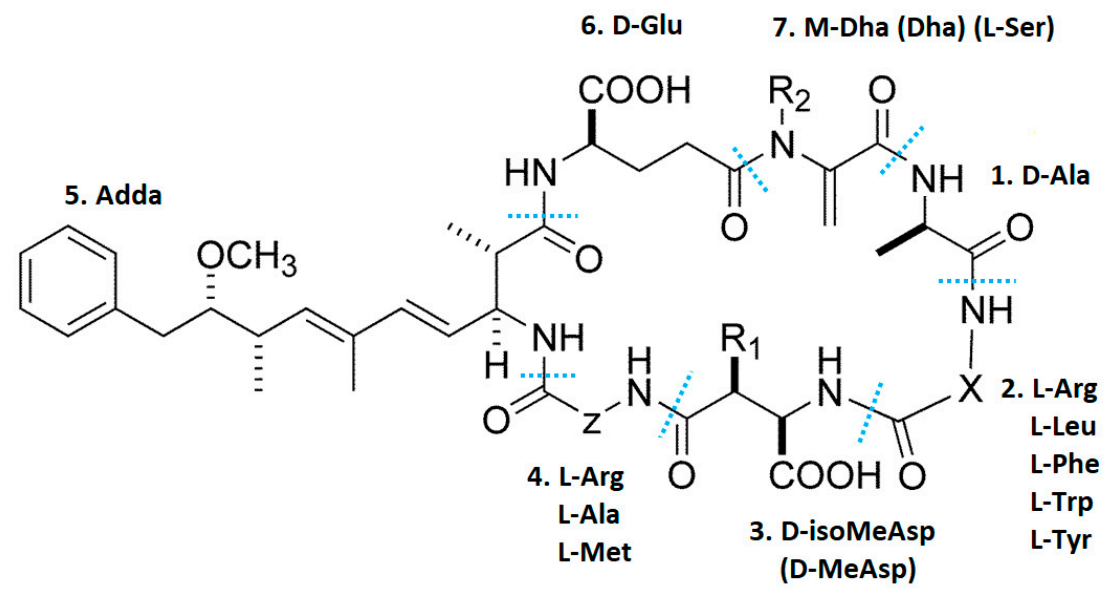

Figure 1. General structure of hepatotoxic cyclic peptides, microcystins. 1-7 signify seven amino acid residues. $\mathrm{X}$ and $\mathrm{Z}$ in positions two and four are highly variable L-amino acids (reproduced from [35], 2019, MDPI).

The cyclic structure of MCs is noted to be responsible for their stability in temperature and $\mathrm{pH}$. MCs are capable to survive at extreme temperatures greater than $300{ }^{\circ} \mathrm{C}$, low temperatures without sunlight and dryness, and extreme high and low $\mathrm{pH}$ [36-38]. MCs are documented to have a size of approximately $3 \mathrm{~nm}$ in diameter and molecular weight ranging between 800 and 1100 Daltons, which primarily depends on the amino acid composition mainly at variable positions and modifications on the incorporated amino acids [39]. Although MCs are known mainly to be polar 
molecules, some variants contain hydrophobic amino acid residues in the highly variable parts of the molecules. For instance, MC-LF and MC-LW, the more hydrophobic phenylalanine (F) and tryptophan (W), respectively, have replaced ' $\mathrm{R}$ ' in MC-LR. The hydrophilic functional groups of MC-LR include carboxyl groups on glutamic and methylaspartic acid and the amino group on ' $R$ ', while the Adda residue is hydrophobic [40]. In addition the net charge of MC-LR was noted to be negative (-1) at most $\mathrm{pH}$ values $(3<\mathrm{pH}<12)$, as the net result of the dissociation of two carboxyl groups and single positive charge of the amino group [41].

\subsection{Toxicity and Carcinogenicity}

Microcystins have shown humans and animals toxicity $[9,21,42,43]$ with lethal dose $\operatorname{LD}_{50}$ by the intraperitoneal route ranging from 50 (MC-LR) to 600 (MC-RR) $\mu \mathrm{g} / \mathrm{kg}$ and oral $\mathrm{LD}_{50}$ of $5000 \mu \mathrm{g} / \mathrm{kg}$ as indicated in mice [8]. The primary mechanism of MCs toxicity is the inhibition of protein phosphatases (PP1) and protein phosphatases (PP2A). This may lead to hyperphosphorylation of key cellular proteins, hepatic hemorrhage, necrosis, inflammation, apoptosis, cytoskeletal and DNA destruction [44,45]. MCs may also induce oxidative stress to trigger cellular apoptosis, destabilize cytoskeleton, enhance cancer cell invasion and damage DNA [46,47].

Microcystins are able to get into the mammalian body through consumption of contaminated water, food and algal dietary supplements, body contact, hemodialysis and in a lesser extent inhalation [9]. Exposure to MCs may show liver toxicity which is the main target organ. Mice exposed to MC-LR had noteworthy rise in clinical chemistry parameters alanine aminotransferase, aspartate aminotransferase, alkaline phosphatase, total bilirubin, and cholesterol, with significant increase in females compared to males. In addition toxic manifestations near the central veins as well as mid lobular areas were observed [48]. At low MC-LR concentration via waterborne exposure, the fish Geophagus brasiliensis exhibited alteration on the liver antioxidant system and histopathologies such as dilation of sinusoids and vacuolization of hepatocytes were evident [49]. Report also indicated that over $80 \%$ of the small population of fishers who lived for many years on Meiliang Bay of Lake Taihu had at least one abnormal serum marker and the serum biochemical indices of liver function including aspartate aminotransferase (AST)/alanine aminotransferase (ALT), triglyceride (TG), globulin (GLB) and lactate dehydrogenase $(\mathrm{LDH})$ revealed liver damage and lipid metabolism dysfunction due to the close positive association with MC contamination [50]. Interestingly the long-term exposure of MC-LR on mice liver also showed hepatic steatosis with molecular alterations in circadian rhythm regulation, lipid metabolic processes, and the cell cycle pathway. Further, at or above the no-observed adverse effect level (NOAEL), MC-LR exposure worsened the pathological phenotype towards nonalcoholic steatohepatitis disease (NASH) or fibrosis [50]. It is worth knowing that other organs including respiratory, renal, cardiovascular, intestinal, central nervous, and reproductive system may also be affected due to the organic anion transporting polypeptides (OATPs) which actively transport MCs into cells $[35,51]$. The chronic low-dose of MC-LR exposure resulted to alveolar collapse, lung cell apoptosis and breach of cell junction integrity. ATII cells were also capable to uptake MC-LR and induced apoptosis and disrupted cell junction integrity [52]. Chronic oral administration of MC-LR also resulted in mitochondrial DNA (mtDNA) neuron damage and histopathological abnormalities as well as mtDNA damage were evident in the hippocampus and cerebral cortex with distinct effects on these two brain regions [53]. Further exposure to MC-LR damaged the microstructure of the jejunum and expression levels of inflammation-related factors interleukin (IL)-1 $\beta$, interleukin (IL)-8, tumor necrosis factor alpha (TNF- $\alpha$ ), as well as transforming growth factor- $\beta 1$ (TGF- $\beta 1$ ), and interleukin (IL)-10 were altered at different MC-LR concentrations [42]. A slight change in serum creatinine (SCr) levels, clear decrease in blood urea nitrogen (BUN) levels, enlarged renal corpuscles and widened of kidney tubules, lymphocyte infiltration in the interstitial tissue, as well as renal pelvis of mice kidney were found after chronic oral MC-LR exposure in mice [54]. In addition, entry of MC-LR in male Macrobrachium rosenbergii testis down-regulated hemolymph testosterone (T) levels, damaged testicular germ cells, mitochondria as well as cell junctions, inhibited testicular development and significantly 
induced the expression of gonadal development related genes in the testis and eyestalk [55]. In zebra fish larvae, MC-LR was noted to cause angiodysplasia, destroy vascular structures and decrease lumen size which triggered a decline of the blood flow area in the blood vessels and brain hemorrhage. Further in the human umbilical vein endothelial cells (HUVECs), varying MC-LR concentrations promoted apoptosis and activated caspase 3/9, increased the level of mitochondrial reactive oxygen species (ROS) and reduced mitochondrial membrane potential. MC-LR also fostered the expression of p53 and inhibited the expression of PCNA [56]. The evidence thus signifies toxic potential of low-dose or chronic exposure to MCs can cause severe chronic injuries or significantly threaten the various organs of mammals and pose potential carcinogenic effects. Therefore people with long-term exposure to MCs may be at a higher risk of developing various diseases including nonalcoholic fatty liver disease (NAFLD), NASH, bronchial tubes, gastrointestinal disorders, inflammatory intestinal disease, Alzheimer disease, acute kidney failure, hypertrophic cardiomyopathy and death.

A number of human and animal fatalities and severe poisonings have been attributed to MC-containing Microcystis blooms $[9,21,57,58]$. MCs were noted to affect the growth and physiological functions of aquatic and terrestrial animals, livestock, pets and wildlife due to their bioaccumulation of these toxins. Moreover MCs were transferred along the food chain and intoxicated other organisms [57]. In Egypt, MCs (free and bound forms) monitored in tilapia fish from three tropical fishponds containing high concentrations of MCs were observed in the tilapia fish intestines, livers and edible tissues, and it was estimated to impose significant negative health consequences on human and other organisms when consumed [59]. A similar investigation was carried out by Greer et al. [60] from aquaculture farms in Southeast Asia and reported the presence of high MCs concentration in the liver and muscle tissue of tilapia fish, which represented a health risk when consumed. Bearing this in mind, further monitoring of MCs, aquaculture farming, fish and beyond is vital to ensure safe water and food for mammalian consumption. In addition, no catching of fish from fishponds during cyanobacterial blooms active safety policies and guidelines to safeguard human health implementations should be ensured and abide. One of the most severe cases of human poisoning occurred in Caruaru, Brazil, in February 1996 when a bloom of Microcystis in a drinking water reservoir contaminated the water supply of a hemodialysis center with MC-LR resulting to 131 patient casualties [58].

It is worth noting that the presence of MCs mainly resulting from irrigation water may also induce plant and crop inhibition of these toxins to reduce yield, poison food and pose high ecological risk. MCs accumulation in roots was found to inhibit growth and further decreased photosynthetic rate and chlorophyll content in rice after irrigation with water contaminated with MCs [61]. The presence of MC-LR and dmMC-LR was noted in fruits of pepper Capsicum annuum and was found to affect antioxidant systems in the fruits after irrigation with contaminated MC water [62]. Further lettuce plants irrigated with MCs contaminated water from the seed and cotyledons stage exhibited higher photosynthetic capacity, chlorophylls as well as leaf nitrogen content, and significant MCs accumulation was observed in various lettuce tissues, constituting a serious public health risk when utilized [63]. Cucumber plants irrigated with MCs extraction contaminated water also inhibited the growth of cucumber at different growth stages (seedling stage $>$ early flowering stage $>$ fruiting stage), and further affected yield and fruit quality. Interestingly contents of vitamin C, soluble sugar and organic acid in fruits of cucumber at seedling stage were declined [64]. It is of interest that irrigation waters containing MCs (MC-LR, MC-RR, and MC-YR) collected from southern China regions were the major source of MCs accumulation in soils and vegetables, and majority of the vegetables exhibited moderate or high human health risk through diet [65]. The findings imply irrigation with MCs contaminated water is capable of threatening plant growth and human health. Based upon this knowledge, it is essential for human to exercise care when ingesting fruits as part of their diet and also strengthen agricultural irrigation management system through monitoring and controlling of contaminated water irrigation with MCs to avoid harmful accumulation of these toxins, destruction to plant growth and potential high ecological and human health hazards. Due to $\mathrm{MC}$ toxic consequences, the International Agency for Research on Cancer (IARC) has categorized this toxin as a possible carcinogen [11]. To 
lessen and prevent MC health hazards, the World Health Organization (WHO) also suggested a provisional $1 \mu \mathrm{g} / \mathrm{L} \mathrm{MC} \mathrm{guidelines} \mathrm{for} \mathrm{drinking} \mathrm{water} \mathrm{quality} \mathrm{[66]} \mathrm{and} \mathrm{maximum} \mathrm{20,000} \mathrm{cyanobacterial}$ cells $\mathrm{mL}^{-1}$ or $10 \mu \mathrm{g} \cdot \mathrm{L}^{-1}$ of chlorophyll-a (where about $2-4 \mu \mathrm{g} \cdot \mathrm{L}^{-1}$ of MCs is expected) guidelines for safe recreational water environment [67].

\section{Treatment Approach}

Microcystins are considered as one of the biggest water pollution problems for global public health due to their ability to induce a range of water safety issues and undesirable health manifestations on animals and humans $[8,9,57,66]$. Drinking water should be safe enough to be utilized with low risk of immediate or long-term harm. In view of this, it is necessary to monitor the utilization of water for toxic cyanobacteria as well as MCs, and treat them for safety domestic, agricultural and recreational purposes. To treat water contaminated with toxin, the toxin's physical and chemical properties, nature as well as cyanobacterial growth and blooms patterns should be considered [8]. A large number of drinking water treatment plants utilize conventional and advanced oxidation water treatment processes to treat water contaminated with MCs. Interestingly, these approaches are sometimes considered too expensive to entirely remove a contaminant that occasionally occurs [68]. Ozonation, chlorination, and chloramination treatment approaches which were unable to thoroughly eliminate MCs in reagent-grade water, Colorado river water and California State Project water further generated by products [69]. The solar/chlorine process, chlorination and solar irradiation though were noted to decrease concentration and hepatotoxicity of MC-LR, complete degradation was not obtained [15]. The ozonation of six MCs also realized a decline in the toxicities and concentrations of MC-LR, MC-RR, MC-LA and MC-LF. Nevertheless, MC-YR and MC-LW revealed a gap between the concentration and toxicity due to partial eradication of Adda [13]. Further conventional coagulation and filtration had limited efficiency in eliminating MCs, as demonstrated in treatment plants located in Czech Republic and in some US states (i.e., California, Texas, Oklahoma, Florida, and Vermont) [70,71]. The findings indicates that the conventional and advanced oxidation water treatment processes are generally expensive to use, ineffective at removing and/or destroying MCs and are capable to generate harmful metabolites. Consequently, it is essential to seek for an efficient and a cost-effective treatment approach that will not invoke any potential harmful metabolites after treatment. The use of bacteria represents a recent investigated and promising biological treatment approach for degrading and removing MCs from water bodies without harming the environment.

\subsection{Biological Degradation by Bacteria}

The biological treatment approach that generally requires little or no maintenance has proven to be environmentally-friendly, effective and can be depended upon to eliminate MCs in water sources compared to the conventional treatment approaches. At present, the biological treatment is becoming more useful as MCs can also be removed without the addition of chemicals capable of producing undesirable metabolites [72-76]. Since the first isolation of bacteria strain capable of degrading MC (from the Murrumbidgee river, Australia [77]), various other bacteria strains have successfully been isolated (Table 1). These bacteria mainly belong to Proteobacteria $(\alpha, \beta$ and $\gamma)$, Actinobacteria and Bacilli. 
Table 1. Microcystin degrading bacteria.

\begin{tabular}{|c|c|c|c|c|c|}
\hline Species & Strain & $m l r$ Gene & Degradable MC Variant & Class Affiliated & Reference \\
\hline Acidovorax facilis & LEw-2 & & MC-LR & $\beta$-proteobacteria & [74] \\
\hline \multirow{2}{*}{ Acinetobacter sp. } & CMDB-2 & & MC-LR & $\gamma$-proteobacteria & [78] \\
\hline & WC-5 & & MC-LR, MC-RR & $\gamma$-proteobacteria & [79] \\
\hline Aeromonas sp. & & & MC-LR & $\gamma$-proteobacteria & [80] \\
\hline \multirow[t]{7}{*}{ Arthrobacter sp. } & C6 & & MC-LR, MC-LW, MC-RR, MC-LF, MC-LY & Actinobacteria & {$[81,82]$} \\
\hline & F7 & & MC-LR, MC-LW, MC-RR, MC-LF, MC-LY & Actinobacteria & {$[81,82]$} \\
\hline & F10 & & MC-LR & Actinobacteria & {$[82]$} \\
\hline & $\mathrm{R} 1$ & & MC-LR & Actinobacteria & [82] \\
\hline & $\mathrm{R} 4$ & & MC-LR, MC-LW, MC-RR, MC-LF, MC-LY & Actinobacteria & {$[81,82]$} \\
\hline & R6 & & MC-LR & Actinobacteria & [82] \\
\hline & R9 & & MC-LR & Actinobacteria & [82] \\
\hline \multirow[t]{5}{*}{ Bacillus sp. } & AMRI-03 & $m \operatorname{lr} A$ & MC-RR & Bacilli & [83] \\
\hline & EMB & $m l r A$ & MC-LR, MC-RR & Bacilli & [84] \\
\hline & JZ-2013 & & MC-LR & Bacilli & [85] \\
\hline & LEw-2010 & & MC-LR & Bacilli & [74] \\
\hline & SSZ01 & $m l r A$ & MC-RR & Bacilli & [86] \\
\hline \multirow[t]{3}{*}{ Bifidobacterium longum } & $\mathrm{Bb} 12$ & & MC-LR & Actinobacteria & [88] \\
\hline & 46 & & MC-LR & Actinobacteria & [88] \\
\hline & 420 & & MC-LR & Actinobacteria & [88] \\
\hline Bordetella sp. & MC-LTH1 & $m l r A$ & MC-LR, MC-RR & $\beta$-proteobacteria & [89] \\
\hline Brevibacillus brevis & LEw-1238 & & MC-LR & Bacilli & [74] \\
\hline Brevibacterium sp. & F3 & & MC-LR, MC-LW, MC-RR, MC-LF, MC-LY & Actinobacteria & [81] \\
\hline Burkholderia sp. & & & MC-LR, $\left[\mathrm{D}-\mathrm{Leu}^{1}\right] \mathrm{MC}-\mathrm{LR}$ & $\beta$-proteobacteria & [90] \\
\hline Enterobacter sp. & YF3 & & MC-LR & $\gamma$-proteobacteria & [72] \\
\hline \multirow[t]{2}{*}{ Lactobacillus rhamnosus } & GG & & MC-LR & Bacilli & [88] \\
\hline & LC-705 & & MC-LR & Bacilli & [88] \\
\hline
\end{tabular}


Table 1. Cont.

\begin{tabular}{|c|c|c|c|c|c|}
\hline Species & Strain & $m l r$ Gene & Degradable MC Variant & Class Affiliated & Reference \\
\hline Lysinibacillus boronitolerans & CQ5 & & MC-LR & Bacilli & [91] \\
\hline Methylobacillus sp. & $\mathrm{J} 10$ & & MC-LR, MC-RR & $\beta$-proteobacteria & [92] \\
\hline \multirow[t]{3}{*}{ Morganella morganii } & C25216 & & MC-LR & Actinobacteria & [93] \\
\hline & C25217 & & MC-LR & Actinobacteria & [93] \\
\hline & $\mathrm{C} 25220$ & & MC-LR & Actinobacteria & [93] \\
\hline \multirow[t]{3}{*}{ Novosphingobium sp. } & KKU-12 & & {$\left[\mathrm{Dha}^{7}\right] \mathrm{MC}-\mathrm{LR}$} & $\alpha$-proteobacteria & [94] \\
\hline & KKU15 & & {$\left[\mathrm{Dha}^{7}\right] \mathrm{MC}-\mathrm{LR}$} & $\alpha$-proteobacteria & [95] \\
\hline & THN-1 & $m \operatorname{lr} A B C D$ & MC-LR & $\alpha$-proteobacteria & [97] \\
\hline Ochrobactrum sp. & FDT5 & & MC-LR & $\alpha$-proteobacteria & [98] \\
\hline \multirow[t]{3}{*}{ Paucibacter sp. } & $\mathrm{CH}$ & & MC-LR & $\beta$-proteobacteria & [99] \\
\hline & IM-4 & & MC-LR, MC-RR, MC-YR & $\beta$-proteobacteria & [100] \\
\hline & $2 \mathrm{C} 20^{\mathrm{T}}$ & & MC-LR, MC-RR, MC-YR & $\beta$-proteobacteria & [101] \\
\hline \multirow[t]{4}{*}{ Pseudomonas sp. } & DMXS & & {$\left[\mathrm{D}-\mathrm{Leu}^{1}\right] \mathrm{MC}-\mathrm{LR}$} & $\gamma$-proteobacteria & [75] \\
\hline & LEw-1033 & & MC-LR & $\gamma$-proteobacteria & [74] \\
\hline & $\begin{array}{c}\text { Pseudomonas } \\
\text { aeruginosa }\end{array}$ & & MC-LR & $\gamma$-proteobacteria & [102] \\
\hline & WC-4 & & MC-LR, MC-RR & $\gamma$-proteobacteria & {$[79]$} \\
\hline Ralstonia solanacearum & & & MC-LR, MC-RR & $\beta$-proteobacteria & [103] \\
\hline Rhizobium sp. & $\mathrm{TH}$ & $m l r A B C D$ & MC-LR & $\alpha$-proteobacteria & [73] \\
\hline \multirow[t]{2}{*}{ Rhodococcus sp. } & $\mathrm{C} 1$ & & MC-LR, MC-LW, MC-RR, MC-LF, MC-LY & Actinobacteria & [81] \\
\hline & $\mathrm{C} 3$ & & MC-LR & Actinobacteria & {$[81,82]$} \\
\hline
\end{tabular}


Table 1. Cont

\begin{tabular}{|c|c|c|c|c|c|}
\hline Species & Strain & mlr Gene & Degradable MC Variant & Class Affiliated & Reference \\
\hline \multirow[t]{6}{*}{ Sphingomonas sp. } & ACM-3962/MJ-PV & $m l r A B C D$ & MC-LR, MC-RR & $\alpha$-proteobacteria & {$[77,104,105]$} \\
\hline & B9 & $m l r A$ & $\begin{array}{c}\text { MC-LR, MC-RR, 3-DMMCLR, DHMCLR, } \\
\text { MCLR-Cys }\end{array}$ & $\alpha$-proteobacteria & [106-108] \\
\hline & MD-1 & $m l r A B C D$ & MC-LR, MC-RR, MC-YR & $\alpha$-proteobacteria & [104] \\
\hline & MDB2 & & & $\alpha$-proteobacteria & [110] \\
\hline & MDB3 & & & $\alpha$-proteobacteria & [110] \\
\hline & $\mathrm{Y} 2$ & $m l r A$ & MC-LR, MC-RR, MC-YR & $\alpha$-proteobacteria & [111] \\
\hline \multirow[t]{10}{*}{ Sphingopyxis sp. } & a7 & $m \operatorname{lr} A C D$ & MC-LR & $\alpha$-proteobacteria & [76] \\
\hline & $\mathrm{C} 1$ & $m l r A B C$ & MC-LR & $\alpha$-proteobacteria & {$[113,114]$} \\
\hline & IM-1 & $m l r A B C D$ & MC-LR, MC-RR, MC-YR & $\alpha$-proteobacteria & [100] \\
\hline & IM-2 & $m l r A B C D$ & MC-LR, MC-RR, MC-YR & $\alpha$-proteobacteria & [100] \\
\hline & IM-3 & $m l r A B C D$ & MC-LR, MC-RR, MC-YR & $\alpha$-proteobacteria & [100] \\
\hline & LH21 & $m l r A B C D$ & MC-LR, MC-LA & $\alpha$-proteobacteria & [115] \\
\hline & m6 & $m l r A B C D$ & MC-LR & $\alpha$-proteobacteria & [116] \\
\hline & USTB05 & $m l r A B C D$ & MC-LR, MC-RR, MC-YR & $\alpha$-proteobacteria & {$[30,118-121]$} \\
\hline & $\mathrm{X} 20$ & $m \operatorname{lr} A B C D$ & MC-LR & $\alpha$-proteobacteria & [122] \\
\hline & YF1 & $m l r A B C D$ & MC-LR & $\alpha$-proteobacteria & [123] \\
\hline \multirow[t]{4}{*}{ Stenotrophomonas sp. } & EMS & $m l r A$ & MC-LR, MC-RR & $\gamma$-proteobacteria & [124] \\
\hline & LEw-1278 & & MC-LR & $\gamma$-proteobacteria & [74] \\
\hline & MC-LTH2 & & MC-LR, MC-RR & $\gamma$-proteobacteria & [125] \\
\hline & $4 \mathrm{~B} 4$ & $m l r A B C D$ & MC-LR, MC-RR, MC-LW, MC-LF & $\gamma$-proteobacteria & [16] \\
\hline
\end{tabular}


Table 1. Cont.

\begin{tabular}{|c|c|c|c|c|c|}
\hline Species & Strain & $m l r$ Gene & Degradable MC Variant & Class Affiliated & Reference \\
\hline $\begin{array}{l}\text { Acinetobacter sp. } \\
\text { Aeromonas sp. } \\
\text { Novosphingobium sp. } \\
\text { Ochrobactrum sp. } \\
\text { Pseudomonas sp. } \\
\text { Rhodococcus sp. } \\
\text { Sphingomonas sp. } \\
\text { Sphingopyxis sp. } \\
\text { Stenotrophomonas sp. } \\
\text { Steroidobacter sp. }\end{array}$ & $\begin{array}{l}\text { Bacterial } \\
\text { consortia }\end{array}$ & & MC-LR & $\begin{array}{c}\gamma \text {-proteobacteria } \\
\gamma \text {-proteobacteria } \\
\alpha \text {-proteobacteria } \\
\alpha \text {-proteobacteria } \\
\gamma \text {-proteobacteria } \\
\text { Actinobacteria } \\
\alpha \text {-proteobacteria } \\
\alpha \text {-proteobacteria } \\
\gamma \text {-proteobacteria } \\
\gamma \text {-proteobacteria }\end{array}$ & [126] \\
\hline $\begin{array}{l}\text { Chryseobacterium sp. } \\
\text { Pseudomonas fragi }\end{array}$ & TSFU & $m l r A B C$ & MC-LR & $\begin{array}{c}\text { Flavobacteriia } \\
\gamma \text {-proteobacteria }\end{array}$ & [127] \\
\hline $\begin{array}{c}\text { Alcaligenes faecalis } \\
\text { Stenotrophomonas } \\
\text { acidaminiohila }\end{array}$ & YFMCD4 & & MC-LR & $\begin{array}{l}\beta \text {-proteobacteria } \\
\gamma \text {-proteobacteria }\end{array}$ & [128] \\
\hline $\begin{array}{c}\text { Klebsiella sp. } \\
\text { Stenotrophomonas sp. }\end{array}$ & YFMCD1 & & MC-LR & $\begin{array}{l}\gamma \text {-proteobacteria } \\
\gamma \text {-proteobacteria }\end{array}$ & [129] \\
\hline $\begin{array}{l}\text { Acinetobacter sp. } \\
\text { Hyphomicrobium aestuarii } \\
\text { Pseudoxanthomonas sp. } \\
\text { Rhizobium sp. } \\
\text { Sphingobium } \mathrm{sp} . \\
\text { Sphingomonas } \mathrm{sp} . \\
\text { Steroidobacter } \mathrm{sp} .\end{array}$ & $\begin{array}{l}\text { Indigenous } \\
\text { bacterial } \\
\text { mixed } \\
\text { culture }\end{array}$ & $m l r A D$ & MC-LR & $\begin{array}{l}\gamma \text {-proteobacteria } \\
\alpha \text {-proteobacteria } \\
\gamma \text {-proteobacteria } \\
\alpha \text {-proteobacteria } \\
\alpha \text {-proteobacteria } \\
\alpha \text {-proteobacteria } \\
\gamma \text {-proteobacteria }\end{array}$ & [130] \\
\hline
\end{tabular}


Table 1. Cont.

\begin{tabular}{|c|c|c|c|c|c|}
\hline Species & Strain & mlr Gene & Degradable MC Variant & Class Affiliated & Reference \\
\hline Agrobacterium sp. & Natural & & MC-LR, Des-MCLR & $\alpha$-proteobacteria & [131] \\
\hline Bosea sp. & bacterial & & & $\alpha$-proteobacteria & \\
\hline Brevundimonas sp. & community & & & $\alpha$-proteobacteria & \\
\hline Rasbo sp. & & & & $\alpha$-proteobacteria & \\
\hline Rhizobium sp. & & & & $\alpha$-proteobacteria & \\
\hline Rhodococcus sp. & & & & Actinobacteria & \\
\hline Roseomonas sp. & & & & $\alpha$-proteobacteria & \\
\hline Nitrosococcus sp. & & & & $\gamma$-proteobacteria & \\
\hline Sandaracinobacter sp. & & & & $\alpha$-proteobacteria & \\
\hline Sphingomonas sp. & & & & $\alpha$-proteobacteria & \\
\hline Bordetella sp. & Bacterial & $m l r A B C D$ & MC-LR & $\beta$-proteobacteria & [132] \\
\hline Burkholderia sp. & community & & & $\beta$-proteobacteria & \\
\hline Cupriavidus sp. & & & & $\beta$-proteobacteria & \\
\hline Methylotenera sp. & & & & $\beta$-proteobacteria & \\
\hline Polaromonas sp. & & & & $\beta$-proteobacteria & \\
\hline Variovorax sp. & & & & $\beta$-proteobacteria & \\
\hline $\begin{array}{l}\text { Microbacterium sp. } \\
\text { Rhizobium sp. }\end{array}$ & $\begin{array}{c}\text { Bacterial } \\
\text { consortium }\end{array}$ & & MC-LR & $\begin{array}{c}\text { Actinobacteria } \\
\alpha \text {-proteobacteria }\end{array}$ & [133] \\
\hline
\end{tabular}

C.: Microcystin; Degradable MC vari 
In practice majority of the MC-degrading bacteria are limited to the genus Sphingomonas [76,104,105]. Other species among the genera Acinetobacter, Arthrobacter, Bacillus, Novosphingobium, Paucibacter, Pseudomonas, Sphingopyxis and Stenotrophomonas have also been reported for MCs degradation (Table 1). Bacillus sp. AMRI-03 completely degraded MC-RR within five days after a lag period of two days [83]. Arthrobacter sp. C6, F7, F10, R1, R4, R6 and R9 demonstrated MC-LR removal within $72 \mathrm{hr}$ [81,82]. Also within 24 days, Pseudomonas aeruginosa DMXS eliminated [D-Leu ${ }^{1}$ ] MC-LR [75]. Novosphingobium sp. KKU-25s also decomposed [Dha(7)]MC-LR within 24 h [96]. Further Stenotrophomonas maltophilia 4B4 showed total MC-LR removed within 10 days while MC-RR and MC-LF elimination occurred within 12 and 14 days respectively [16]. In a recent publication, a novel bacteria strain YF1 of the genus Sphingopyxis indicated thorough MC-LR degradation within 120 min [123]. The evidence thus indicates that bacteria strains isolated from many different environmental habitats around the world have strong MC-degrading ability, and may play a significant role in the natural degradation and removal of MCs.

The combination of two or more bacteria strains (bacterial community) have also proven to be capable of degrading MC-LR (Table 1). The seven isolates Acinetobacter sp., Hyphomicrobium aestuarii, Pseudoxanthomonas sp., Rhizobium sp., Sphingobium sp., Sphingomonas sp. and Steroidobacter sp. from Taiwan reservoir, China [130] and 10 isolates Acinetobacter sp., Aeromonas sp., Novosphingobium sp., Ochrobactrum sp., Pseudomonas sp., Rhodococcus sp., Sphingomonas sp., Sphingopyxis sp., Stenotrophomonas sp. and Steroidobacter sp. from drinking water reservoir in Southern California [126] successfully indicated complete MC-LR degradation. Moreover bacterial community isolated from natural waters with previous cyanobacterial contamination was noted to entirely removal MC-LR without lag phase, however, the composition of the bacterial community was not analyzed [134]. In a recent study, acclimatized-TSFU bacterial community comprising of novel strains Chryseobacterium sp. and Pseudomonas fragi was also found to completely degrade MC-LR [127]. The natural bacterial community mainly consisting of Agrobacterium sp., Bosea sp., Brevundimonas sp., Hyphomicrobium sp., Rasbo sp., Rhizobium sp., Rhodococcus sp., Roseomonas sp., Mesorhizobium sp., Nitrosococcus sp., Sandaracinobacter sp. and Sphingomonas sp. isolated from the mucilage of M. aeruginosa colonies during a bloom in a French pond completely degraded MC-LR and Des-MCLR. It is of interest that the bacterial community also degraded cyanobacterial secondary metabolites such as cyanopeptolins and aerucyclamides [131]. The findings of this study suggest that, bacterial community may possess the ability not only to degrade $\mathrm{MC}$ but also other cyanobacterial secondary metabolites. At present only a few MC-degrading bacterial communities have been obtained for MC-LR degradation. Further studies are required to investigate the degrading ability of bacterial community on other MC variants.

\subsection{Enzymatic Mechanisms of Microcystins Biodegradation}

A growing number of novel bacteria strains with the ability to degrade MCs are being revealed. However, only one metabolic pathway liable for degrading these toxins and is encoded by the $m l r$ gene cluster is fully described. A novel pathway involving four genes ( $m l r A B C D)$, three intracellular hydrolytic enzymes (MlrABC) and two intermediate products; linearized MC-LR (Adda-Glu-Mdha-Ala-Leu-Masp-Arg-OH) and tetrapeptide (Adda-Glu-Mdha-Ala-OH) for MC-LR degradation using Sphingomonas sp. ACM- 3962 was demonstrated [105]. The MlrA enzyme encoded by $m l r A$ gene cleaved the Adda-Arg peptide bond of MC-LR, which converted MC-LR to linearized MC-LR. The MlrB enzyme encoded by mlrB gene hydrolyzed the Ala-Leu bond, converting the linearized MC-LR to a tetrapeptide. The MlrC enzyme encoded by $m l r C$ gene broke the tetrapeptide into smaller peptides and amino acids. Transport of $\mathrm{MC}$ and its degradation products were assumed to be facilitated via $m l r D$ gene [135]. Nevertheless, these smaller peptides and amino acids were not well described. In subsequent investigations, Imanishi et al. [106] and Harada et al. [107] showed products of MC-LR degradation which were consistent with the previous report $[105,135]$ and further isolated Adda using Sphingomonas sp. B-9. Successive studies have also confirmed the existence of $m l r$ cluster components in other MC-degrading bacteria species and strains (as depicted in Table 1). 
Regarding the sequential enzymatic hydrolyses of peptide bonds, $m l r C$ gene was found not only to act on the tetrapeptide but is also capable to hydrolyze linearized MC-LR without earlier processing by $m l r B$ gene $[114,136]$. Sphingopxyis sp. USTB-05 showed complete MCs (MC-LR, MC-RR and MC-YR) degradation with different intermediate products [30,118-121]. These findings suggest that the enzymatic processes and degradation pathways may vary between MC variants and bacteria strains. Further aside the known MC-LR degradation products (linearized MC-LR, tetrapeptide and Adda), Ding et al. [116] identified eight new different intermediate degradation products namely, three tripeptides (Adda-Glu-Mdha, Glu-Mdha-Ala, and Leu-MeAsp-Arg), three dipeptides (Glu-Mdha, Mdha-Ala, and MeAsp-Arg) and two amino acids (Leu, and Arg) using Sphingopxyis sp. m6. These findings also suggest that more than three intermediate products are capable to occur during MC degradation. In a recent study Yang et al. [123] found a potential link between paa gene clusters for phenylacetic acid (PAA) degradation in the neighborhoods of $m l r A B C D$ gene cluster. Interestingly complete MC-LR degradation by Sphingopyxis sp. YF1 without Adda as the final degradation product (as illustrated in Figure 2.) was noted. MC-LR was sequentially degraded into linearized MC-LR, tetrapeptide and Adda. The Adda was further degraded into PAA, and was converted into PAA-CoA by PAAase. Subsequently PAA-CoA was degraded to acetyl coenzyme A (acetyl-CoA) by PaaA, PaaG and PaaZ homologues. Finally, acetyl-CoA was completely converted to $\mathrm{CO}_{2}$ through tricarboxylic acid (TCA) cycle [123]. The evidence suggests that PAA may be the downstream metabolism path of MC degradation. However, further study needs to determine the kind of enzyme that degraded Adda into PAA.

It is well established that the degradation pathways and enzymatic processes in $\alpha, \beta$ and $\gamma$ Proteobacteria strains among the genera Bordetella, Sphingomonas, Sphingopxyis Stentophomonas and Rhizobium $[17,73,89,104,106,111,113,115,117,124]$ as well as bacterial communities including Acinetobacter sp., Hyphomicrobium aestuarii, Pseudoxanthomonas sp., Rhizobium sp., Sphingobium sp., Sphingomonas sp. and Steroidobacter sp. (Indigenous bacterial mixed culture) and Chryseobacterium sp. and Pseudomonas fragi (Acclimatized-TSFU) $[127,130]$ are well described. These bacteria strains and bacterial communities found to degrade different variants of MC contained the $m l r$ homologues genes, and linearized MC, tetrapeptide and/or Adda were evident as intermediate products. At present only the $m l r A$ gene has been observed in Bacillus sp. $[83,84,86]$. Although the Bacillus sp., Paucibacter sp., Pseudomonas sp. and Ralstonia solanacearum as well as bacterial communities including Klebsiella sp. and Stenotrophomonas sp. (YFMCD1), Alcaligenes faecalis and Stenotrophomonas acidaminiohila (YFMCD4) and Bordetella sp., Burkholderia sp., Cupriavidus sp., Methylotenera sp., Polaromonas sp., Polynucleobacter sp., Ralstonia sp. and Variovorax sp. (Bacterial community) (as depicted in Table 1) also degraded different variants of MC, the degradation pathways and/or enzymatic processes were inadequately described. Clearly, further investigation needs to elucidate the MC-degrading metabolism of these strains and communities. It is of interest that the MC degradation mechanism for Acinetobacter sp., Aeromonas sp., Arthrobacter sp., Bifidobacterium longum, Brevibacterium sp., Burkholderia sp., Lactobacillus rhamnosus, Methylobacillus sp., Morganella morganii, Ochrobactrum sp. and Rhodococous sp. as well as bacterial communities including Acinetobacter sp., Aeromonas sp., Novosphingobium sp., Ochrobactrum sp., Pseudomonas sp., Rhodococcus sp., Sphingomonas sp., Sphingopyxis sp., Stenotrophomonas sp. and Steroidobacter (Bacterial consortia) and Microbacterium sp. and Rhizobium sp. (Bacterial consortium) (as shown in Table 1) also need to be identified and clarified. It is worth noting that the Enterobacter sp. YF3, Acidovorax facilis Lew-2, Bacillus thuringiensis LEw-2010, Brevibacillus brevis LEw-1238, Pseudomonas putida LEw-1033 and LEw-2166, Stenotrophomonas maltophila LEw-1278 and Bacillus sp. [72,74,87] demonstrated MC-LR degradation independent of the MC-degrading $m \operatorname{lr} A B C D$ genes. These findings indicate that MC degradation may not necessarily follow the pathways of the $m l r$ degrading mechanism. 


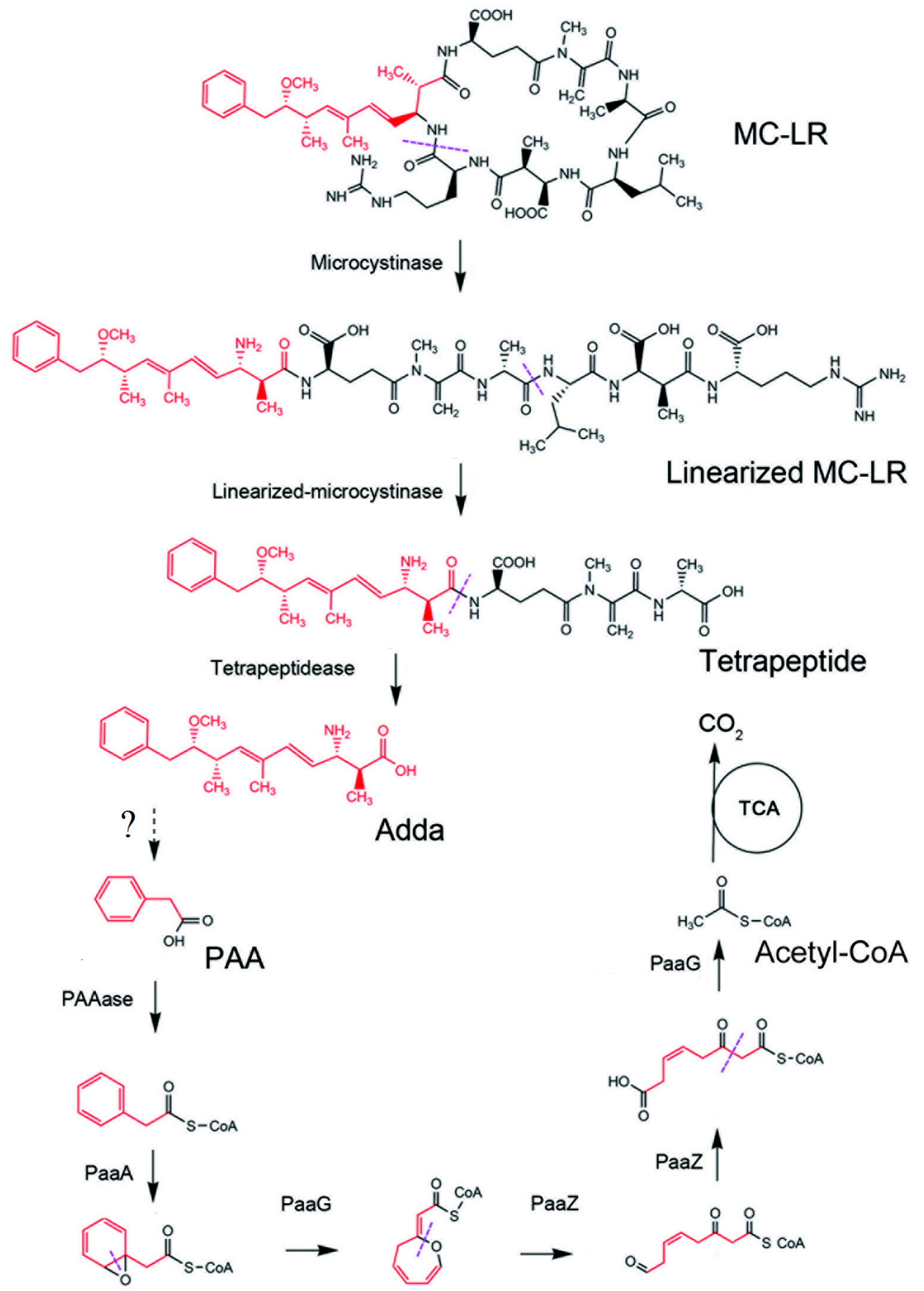

Figure 2. Biodegradation pathway of MC-LR by Sphingopyxis sp. YF1 (reproduced from [123], 2020, Elsevier Ltd.). 


\section{Conclusions}

In this current paper, toxic effects and bacterial degradation of MCs were reviewed. Studies indicate that the cyclic heptapeptide hepatotoxins MCs are primarily produced by species of Microcystis containing a series of MC synthesis genes. MCs inhibit protein phosphatases and constitute a natural health hazards in the environment. MCs are chemically stable in water and conventional water treatment approaches have failed to completely remove them. MC toxic effect led to the establishment of WHO's $1 \mu \mathrm{g} / \mathrm{L}$ MC guidelines for safe drinking water quality. To provide safe drinking water, biological degradation is considered the most successful solution for MCs removal in the natural environment. Bacteria identification for different variants of MC degradation has been reported from various environmental habitats worldwide. The metabolism of these bacteria showed four genes $(m l r A B C D)$, three intracellular hydrolytic enzymes (MlrABC) and three degrading products (linearized MC-LR, tetrapeptide and Adda). However studies about Sphingopxyis that resulted to a hydrolytic pathway via the discovery of eight new intermediate products, identification of different intermediate products from varying MC variants and the downstream MC degradation route by PAA metabolism which identified $\mathrm{CO}_{2}$ as the final degrading product have paved way for additional investigations on the biodegradation mechanism of MCs. Further studies need to explore the PAA metabolism on other MC degrading bacteria strains. Moreover, whether any degradation products exist between Adda and PAA, how the degrading product can be converted into PAA, and the functional genes as well as proteins involved in the metabolism process, need to be determined.

Author Contributions: F.Y. and I.Y.M. conceived the manuscript; I.Y.M. wrote the paper. All the authors participated in the preparation of the manuscript. All authors have read and agreed to the published version of the manuscript.

Funding: This work was supported by the National Natural Science Foundation $(81773393,81502787)$; Central South University Innovation Driven Project (20170027010004).

Conflicts of Interest: The authors declare no conflict of interest.

\section{References}

1. WHO. Technical Brief Technical Management of Cyanobacteria in Drinkingwater Supplies: Information for Regulators and Water Suppliers; World Health Organization: Geneva, Switzerland, 2011.

2. Ji, X.; Verspagen, J.M.H.; Stomp, M.; Huisman, J. Competition between cyanobacteria and green algae at low versus elevated $\mathrm{CO}_{2}$ : Who will win, and why? J. Exp. Bot. 2017, 68, 3815-3828. [CrossRef] [PubMed]

3. Jia, Y.; Chen, W.; Zuo, Y.; Lin, L.; Song, L. Heavy metal migration and risk transference associated with cyanobacterial blooms in eutrophic freshwater. Sci. Total Environ. 2018, 613-614, 1324-1330. [CrossRef] [PubMed]

4. Silveira, S.B.; Odebrecht, C. Effects of Salinity and temperature on the growth, toxin production, and akinete germination of the cyanobacterium Nodularia spumigena. Front. Mar. Sci. 2019, 6. [CrossRef]

5. Wang, J.H.; Yang, C.; He, L.Q.; Dao, G.H.; Du, J.S.; Han, Y.P.; Wu, G.X.; Wu, Q.Y.; Hu, H.Y. Meteorological factors and water quality changes of Plateau Lake Dianchi in China (1990-2015) and their joint influences on cyanobacterial blooms. Sci. Total Environ. 2019, 665, 406-418. [CrossRef]

6. Jankowiak, J.; Hattenrath-Lehmann, T.; Kramer, B.J.; Ladds, M.; Gobler, C.J. Deciphering the effects of nitrogen, phosphorus, and temperature on cyanobacterial bloom intensification, diversity, and toxicity in western Lake Erie. Limnol. Oceanogr. 2019, 64, 1347-1370. [CrossRef]

7. Drobac, D.; Tokodi, N.; Simeunovic, J.; Baltic, V.; Stanic, D.; Svircev, Z. Human exposure to cyanotoxins and their effects on health. Arh. Hig. Rada Toksikol. 2013, 64, 119-130. [CrossRef]

8. Sivonen, K.; Jones, G. Cyanobacterial toxins. In Toxic Cyanobacteria in Water. A Guide to Their Public Health Consequences, Monitoring and Management; Chorus, I., Bartram, J., Eds.; E and FN Spon: London, UK, 1999; pp. 41-111.

9. Massey, I.Y.; Yang, F.; Ding, Z.; Yang, S.; Guo, J.; Tezi, C.; Al-Osman, M.; Kamegni, R.B.; Zeng, W. Exposure routes and health effects of microcystins on animals and humans: A mini-review. Toxicon 2018, 151, 156-162. [CrossRef] 
10. Zhang, C.; Massey, I.Y.; Liu, Y.; Huang, F.; Gao, R.; Ding, M.; Xiang, L.; He, C.; Wei, J.; Li, Y.; et al. Identification and characterization of a novel indigenous algicidal bacterium Chryseobacterium species against Microcystis aeruginosa. J. Toxicol. Environ. Health Part A 2019, 1-9. [CrossRef] [PubMed]

11. IARC. Ingested Nitrate and Nitrite, and Cyanobacterial Peptide Toxins; World Health Organization, International Agency for Research on Cancer: Lyon, France, 2010.

12. Mantzouki, E.; Lürling, M.; Fastner, J.; de Senerpont Domis, L.; Wilk-Woźniak, E.; Koreivienė, J.; Seelen, L.; Teurlincx, S.; Verstijnen, Y.; Krztoń, W.; et al. Temperature Effects Explain Continental Scale Distribution of Cyanobacterial Toxins. Toxins 2018, 10, 156. [CrossRef]

13. Kim, M.S.; Lee, C. Ozonation of Microcystins: Kinetics and Toxicity Decrease. Environ. Sci. Technol. 2019, 53, 6427-6435. [CrossRef]

14. Massey, I.Y.; Zhang, X.; Yang, F. Importance of bacterial biodegradation and detoxification processes of microcystins for environmental health. J. Toxicol. Environ. Health Part B 2018, 1-13. [CrossRef]

15. Sun, J.L.; Bu, L.J.; Chen, S.Y.; Lu, X.L.; Wu, Y.T.; Shi, Z.; Zhou, S.Q. Oxidation of Microcystin-LR via the solar/chlorine process: Radical mechanism, pathways and toxicity assessment. Ecotoxicol. Environ. Saf. 2019, 183. [CrossRef]

16. Idroos, E.S.; De Silva, B.; Manage, P.M. Biodegradation of microcystin analogues by Stenotrophomonas maltophilia isolated from Beira Lake Sri Lanka. J. Natl. Sci. Found. Sri Lanka 2017, 45, 91-99. [CrossRef]

17. Maghsoudi, E.; Fortin, N.; Greer, C.; Maynard, C.; Page, A.; Duy, S.V.; Sauve, S.; Prevost, M.; Dorner, S. Cyanotoxin degradation activity and $m l r$ gene expression profiles of a Sphingopyxis sp. isolated from Lake Champlain, Canada. Environ. Sci. Process. Impacts 2016, 18, 1417-1426. [CrossRef] [PubMed]

18. Liu, P.; Wei, J.; Yang, K.; Massey, I.Y.; Guo, J.; Zhang, C.; Yang, F. Isolation, molecular identification, and characterization of a unique toxic cyanobacterium Microcystis sp. found in Hunan Province, China. J. Toxicol. Environ. Health Part A 2018, 81, 1142-1149. [CrossRef] [PubMed]

19. Wei, J.; Xie, X.; Huang, F.; Xiang, L.; Wang, Y.; Han, T.; Massey, I.Y.; Liang, G.; Pu, Y.; Yang, F. Simultaneous Microcystis algicidal and microcystin synthesis inhibition by a red pigment prodigiosin. Environ. Pollut. 2019, 256, 113444. [CrossRef]

20. Metcalf, J.S.; Richer, R.; Cox, P.A.; Codd, G.A. Cyanotoxins in desert environments may present a risk to human health. Sci. Total Environ. 2012, 421-422, 118-123. [CrossRef]

21. Svircev, Z.; Lalic, D.; Bojadzija, S.G.; Tokodi, N.; Drobac, B.D.; Chen, L.; Meriluoto, J.; Codd, G.A. Global geographical and historical overview of cyanotoxin distribution and cyanobacterial poisonings. Arch. Toxicol. 2019. [CrossRef]

22. Zhang, X.J.; Chen, C.; Ding, J.Q.; Hou, A.; Li, Y.; Niu, Z.B.; Su, X.Y.; Xu, Y.J.; Laws, E.A. The 2007 water crisis in Wuxi, China: Analysis of the origin. J. Hazard. Mater. 2010, 182, 130-135. [CrossRef]

23. Harke, M.J.; Steffen, M.M.; Gobler, C.J.; Otten, T.G.; Wilhelm, S.W.; Wood, S.A.; Paerl, H.W. A review of the global ecology, genomics, and biogeography of the toxic cyanobacterium, Microcystis spp. Harmful Algae 2016, 54, 4-20. [CrossRef]

24. LeBlanc, P.; Merkley, N.; Thomas, K.; Lewis, N.I.; Bekri, K.; Renaud, S.L.; Pick, F.R.; McCarron, P.; Miles, C.O.; Quilliam, M.A. Isolation and Characterization of DLeu1 microcystin-LY from Microcystis aeruginosa CPCC-464. Toxins 2020, 12, 77. [CrossRef] [PubMed]

25. Feng, H.; Clara, T.; Huang, F.Y.; Wei, J.; Yang, F. Identification and characterization of the dominant Microcystis sp. cyanobacteria detected in Lake Dong Ting, China. J. Toxicol. Environ. Health Part A Curr. Issues 2019, 82, 1143-1150. [CrossRef] [PubMed]

26. Gkelis, S.; Tussy, P.F.; Zaoutsos, N. Isolation and preliminary characterization of cyanobacteria strains from freshwaters of Greece. Open Life Sci. 2015, 10, 52-60. [CrossRef]

27. Tillett, D.; Dittmann, E.; Erhard, M.; von Dohren, H.; Borner, T.; Neilan, B.A. Structural organization of microcystin biosynthesis in Microcystis aeruginosa PCC7806: An integrated peptide-polyketide synthetase system. Chem. Biol. 2000, 7, 753-764. [CrossRef]

28. Carmichael, W.W. The cyanotoxins. Adv. Bot. Res. 1997, 27, 211-256.

29. Carmichael, W.W. Cyanobacteria secondary metabolites-the cyanotoxins. J. Appl. Bacteriol. 1992, 72, 445-459. [CrossRef]

30. Zhang, M.; Pan, G.; Yan, H. Microbial biodegradation of microcystin-RR by bacterium Sphingopyxis sp. USTB-05. J. Environ. Sci. 2010, 22, 168-175. [CrossRef] 
31. Spoof, L.; Catherine, A. Appendix 3 Tables of Microcystins and Nodularins. Handbook of Cyanobacterial Mmonitoring and Cyanotoxin Analysis; John Wiley \& Sons, Ltd.: Hoboken, NJ, USA, 2017; pp. 526-537. [CrossRef]

32. Spoof, L.; Vesterkvist, P.; Lindholm, T.; Meriluoto, J. Screening for cyanobacterial hepatotoxins, microcystins and nodularin in environmental water samples by reversed-phase liquid chromatography-electrospray ionisation mass spectrometry. J. Chromatogr. A 2003, 1020, 105-119. [CrossRef]

33. Gurbuz, F.; Metcalf, J.S.; Karahan, A.G.; Codd, G.A. Analysis of dissolved microcystins in surface water samples from Kovada Lake, Turkey. Sci. Total Environ. 2009, 407, 4038-4046. [CrossRef] [PubMed]

34. Bittencourt-Oliveira, M.C.; Oliveira, M.C.; Pinto, E. Diversity of microcystin-producing genotypes in Brazilian strains of Microcystis (Cyanobacteria). Braz. J. Biol. 2011, 71, 209-216. [CrossRef]

35. Cao, L.; Massey, I.Y.; Feng, H.; Yang, F. A Review of Cardiovascular Toxicity of Microcystins. Toxins 2019, 11, 507. [CrossRef] [PubMed]

36. Tsuji, K.; Naito, S.; Kondo, F.; Ishikawa, N.; Watanabe, M.F.; Suzuki, M.; Harada, K. Stability of microcystins from cyanobacteria: Effect of light on decomposition and isomerization. Environ. Sci. Technol. 2004, 28, 173-177. [CrossRef] [PubMed]

37. De Figueiredo, D.R.; Azeiteiro, U.M.; Esteves, S.M.; Goncalves, F.J.; Pereira, M.J. Microcystin-producing blooms-a serious global public health issue. Ecotoxicol. Environ. Saf. 2004, 59, 151-163. [CrossRef] [PubMed]

38. Harada, K.; Tsuji, K.; Watanabe, M.F.; Kondo, F. Stability of microcystins from cyanobacteria-III. Effect of pH and temperature. Phycologia 1996, 35, 83-88. [CrossRef]

39. Donati, C.; Drikas, M.; Hayes, R.; Newcombe, G. Microcystin-LR adsorption by powdered activated carbon. Water Res. 1994, 28, 1735-1742. [CrossRef]

40. Vesterkvist, P.S.M.; Misiorek, J.O.; Spoof, L.E.M.; Toivola, D.M.; Meriluoto, J.A.O. Comparative Cellular Toxicity of Hydrophilic and Hydrophobic Microcystins on Caco-2 Cells. Toxins 2012, 4, 1008-1023. [CrossRef]

41. Feitz, A.J.; Waite, T.D.; Jones, G.J.; Boyden, B.H.; Orr, P.T. Photocatalytic degradation of the blue green algal toxin microcystin-LR in a natural organic-aqueous matrix. Environ. Sci. Technol. 1999, 33, 243-249. [CrossRef]

42. Cao, L.; Huang, F.; Massey, I.Y.; Wen, C.; Zheng, S.; Xu, S.; Yang, F. Effects of Microcystin-LR on the Microstructure and Inflammation-Related Factors of Jejunum in Mice. Toxins 2019, 11, 482. [CrossRef]

43. Alosman, M.; Cao, L.H.; Massey, I.Y.; Yang, F. The lethal effects and determinants of microcystin-LR on heart: A mini review. Toxin Rev. 2020. [CrossRef]

44. MacKintosh, C.; Beattie, K.A.; Klumpp, S.; Cohen, P.; Codd, G.A. Cyanobacterial microcystin-LR is a potent and specific inhibitor of protein phosphatases 1 and 2A from both mammals and higher plants. FEBS Lett. 1990, 264, 187-192. [CrossRef]

45. Dawson, R.M. The toxicology of microcystins. Toxicon 1998, 36, 953-962. [CrossRef]

46. Zhou, M.; Tu, W.; Xu, J. Mechanisms of microcystin-LR-induced cytoskeletal disruption in animal cells. Toxicon 2015, 101, 92-100. [CrossRef] [PubMed]

47. Liu, J.; Sun, Y. The role of PP2A-associated proteins and signal pathways in microcystin-LR toxicity. Toxicol. Lett. 2015, 236, 1-7. [CrossRef] [PubMed]

48. Mrdjen, I.; Morse, M.A.; Ruch, R.J.; Knobloch, T.J.; Choudhary, S.; Weghorst, C.M.; Lee, J. Impact of Microcystin-LR on Liver Function Varies by Dose and Sex in Mice. Toxins 2018, 10, 435. [CrossRef] [PubMed]

49. Calado, S.L.D.; Vicentini, M.; Santos, G.S.; Pelanda, A.; Santos, H.; Coral, L.A.; Magalhaes, V.D.; Mela, M.; Cestari, M.M.; de Assis, H.C.S. Sublethal effects of microcystin-LR in the exposure and depuration time in a neotropical fish: Multibiomarker approach. Ecotoxicol. Environ. Saf. 2019, 183. [CrossRef] [PubMed]

50. Zhao, Y.; Yan, Y.; Xie, L.; Wang, L.; He, Y.; Wan, X.; Xue, Q. Long-term environmental exposure to microcystins increases the risk of nonalcoholic fatty liver disease in humans: A combined fisher-based investigation and murine model study. Environ. Int. 2020, 138, 105648. [CrossRef] [PubMed]

51. Chen, L.; Xie, P. Mechanisms of microcystin-induced cytotoxicity and apoptosis. Mini Rev. Med. Chem. 2016, 16, 1018-1031. [CrossRef]

52. Wang, C.; Gu, S.; Yin, X.; Yuan, M.; Xiang, Z.; Li, Z.; Cao, H.; Meng, X.; Hu, K.; Han, X. The toxic effects of microcystin-LR on mouse lungs and alveolar type II epithelial cells. Toxicon 2016, 115, 81-88. [CrossRef]

53. Wang, X.F.; Xu, L.Z.; Li, X.X.; Chen, J.W.; Zhou, W.; Sun, J.P.; Wang, Y.P. The differential effects of microcystin-LR on mitochondrial DNA in the hippocampus and cerebral cortex. Environ. Pollut. 2018, 240, 68-76. [CrossRef] 
54. Yi, X.P.; Xu, S.S.; Huang, F.Y.; Wen, C.; Zheng, S.L.; Feng, H.; Guo, J.; Chen, J.H.; Feng, X.L.; Yang, F. Effects of chronic exposure to microcystin-LR on kidney in mice. Int. J. Environ. Res. Public Health 2019, 16, 5030. [CrossRef]

55. Zhang, Y.Y.; Zhuang, H.; Yang, H.; Xue, W.; Wang, L.F.; Wei, W.Z. Microcystin-LR disturbs testicular development of giant freshwater prawn Macrobrachium rosenbergii. Chemosphere 2019, 222, 584-592. [CrossRef] [PubMed]

56. Wang, Q.L.; Liu, Y.L.; Guo, J.S.; Lin, S.; Wang, Y.Q.; Yin, T.Y.; Gregersen, H.; Hu, T.Z.; Wang, G.X. Microcystin-LR induces angiodysplasia and vascular dysfunction through promoting cell apoptosis by the mitochondrial signaling pathway. Chemosphere 2019, 218, 438-448. [CrossRef]

57. Rastogi, R.P.; Sinha, R.P.; Incharoensakdi, A. The cyanotoxin-microcystins: Current overview. Rev. Environ. Sci. Bio Technol. 2014, 13, 215-249. [CrossRef]

58. Jochimsen, E.M.; Carmichael, W.W.; An, J.S.; Cardo, D.M.; Cookson, S.T.; Holmes, C.E.; Antunes, M.B.; de Melo Filho, D.A.; Lyra, T.M.; Barreto, V.S.; et al. Liver failure and death after exposure to microcystins at a hemodialysis center in Brazil. N. Engl. J. Med. 1998, 338, 873-878. [CrossRef] [PubMed]

59. Mohamed, Z.; Ahmed, Z.; Bakr, A.; Hashem, M.; Alamri, S. Detection of free and bound microcystins in tilapia fish from Egyptian fishpond farms and its related public health risk assessment. J. Consum. Prot. Food Saf. 2020, 15, 37-47. [CrossRef]

60. Greer, B.; Maul, R.; Campbell, K.; Elliott, C. Detection of freshwater cyanotoxins and measurement of masked microcystins in tilapia from Southeast Asian aquaculture farms. Anal. Bioanal. Chem. 2017, 409, 4057-4069. [CrossRef] [PubMed]

61. Liang, C.; Wang, W. Response and recovery of rice (Oryza sativa) seedlings to irrigation with microcystin-contaminated water. Environ. Earth Sci. 2015, 73, 4573-4580. [CrossRef]

62. Drobac, D.; Tokodi, N.; Kiprovski, B.; Malencic, D.; Vazic, T.; Nybom, S.; Meriluoto, J.; Svircev, Z. Microcystin accumulation and potential effects on antioxidant capacity of leaves and fruits of Capsicum annuum. J. Toxicol. Environ. Health Part A Curr. Issues 2017, 80, 145-154. [CrossRef]

63. Levizou, E.; Statiris, G.; Papadimitriou, T.; Laspidou, C.S.; Kormas, K.A. Lettuce facing microcystins-rich irrigation water at different developmental stages: Effects on plant performance and microcystins bioaccumulation. Ecotoxicol. Environ. Saf. 2017, 143, 193-200. [CrossRef]

64. Zhu, J.Z.; Ren, X.Q.; Liu, H.Y.; Liang, C.J. Effect of irrigation with microcystins-contaminated water on growth and fruit quality of Cucumis sativus L. and the health risk. Agric. Water Manag. 2018, 204, 91-99. [CrossRef]

65. Xiang, L.; Li, Y.W.; Liu, B.L.; Zhao, H.M.; Li, H.; Cai, Q.Y.; Mo, C.H.; Wong, M.H.; Li, Q.X. High ecological and human health risks from microcystins in vegetable fields in southern China. Environ. Int. 2019, 133. [CrossRef] [PubMed]

66. WHO. Cyanobacterial Toxins: Microcystin-LR. Guidelines for Drinking Water Quality; World Health Organization: Geneva, Switzerland, 1998.

67. WHO. Coastal and Fresh Waters, Guidelines for Safe Recreational Water Environments; World Health Organization: Geneva, Switzerland, 2003.

68. He, X.; Liu, Y.L.; Conklin, A.; Westrick, J.; Weavers, L.K.; Dionysiou, D.D.; Lenhart, J.J.; Mouser, P.J.; Szlag, D.; Walker, H.W. Toxic cyanobacteria and drinking water: Impacts, detection, and treatment. Harmful Algae 2016, 54, 174-193. [CrossRef] [PubMed]

69. Guo, Y.C.B.; Lee, A.K.; Yates, R.S.; Liang, S.; Rochelle, P.A. Analysis of Microcystins in Drinking Water by ELISA and LC/MS/MS. J. Am. Water Works Assoc. 2017, 109, 13-25. [CrossRef]

70. Jasa, L.; Sadilek, J.; Kohoutek, J.; Strakova, L.; Marsalek, B.; Babica, P. Application of passive sampling for sensitive time-integrative monitoring of cyanobacterial toxins microcystins in drinking water treatment plants. Water Res. 2019, 153, 108-120. [CrossRef]

71. Szlag, D.C.; Sinclair, J.L.; Southwell, B.; Westrick, J.A. Cyanobacteria and Cyanotoxins Occurrence and Removal from Five High-Risk Conventional Treatment Drinking Water Plants. Toxins 2015, 7, 2198-2220. [CrossRef]

72. Huang, F.Y.; Feng, H.; Li, X.Y.; Yi, X.P.; Guo, J.; Clara, T.; Yang, F. Anaerobic degradation of microcystin-LR by an indigenous bacterial Enterobacter sp. YF3. J. Toxicol. Environ. Health Part A Curr. Issues 2019. [CrossRef] 
73. Zhu, X.Y.; Shen, Y.T.; Chen, X.G.; Hu, Y.O.O.; Xiang, H.Y.; Tao, J.; Ling, Y. Biodegradation mechanism of microcystin-LR by a novel isolate of Rhizobium sp. TH and the evolutionary origin of the mlrA gene. Int. Biodeterior. Biodegrad. 2016, 115, 17-25. [CrossRef]

74. Krishnan, A.; Zhang, Y.Q.; Mou, X.Z. Isolation and Characterization of Microcystin-Degrading Bacteria from Lake Erie. Bull. Environ. Contam. Toxicol. 2018, 101, 617-623. [CrossRef]

75. Lemes, G.A.; Kist, L.W.; Bogo, M.R.; Yunes, J.S. Biodegradation of [D-Leu(1)] microcystin-LR by a bacterium isolated from sediment of Patos Lagoon estuary, Brazil. J. Venom. Anim. Toxins Incl. Trop. Dis. 2015, $21,4$. [CrossRef]

76. Zhang, J.; Lu, Q.; Ding, Q.; Yin, L.; Pu, Y. A Novel and Native Microcystin-Degrading Bacterium of Sphingopyxis sp. Isolated from Lake Taihu. Int. J. Environ. Res. Public Health 2017, 14, 1187. [CrossRef]

77. Jones, G.J.; Bourne, D.G.; Blakeley, R.L.; Doelle, H. Degradation of the cyanobacterial hepatotoxin microcystin by aquatic bacteria. Nat. Toxins 1994, 2, 228-235. [CrossRef] [PubMed]

78. Li, H.; Ai, H.; Kang, L.; Sun, X.; He, Q. Simultaneous Microcystis Algicidal and Microcystin Degrading Capability by a Single Acinetobacter Bacterial Strain. Environ. Sci. Technol. 2016, 50, 11903-11911. [CrossRef] [PubMed]

79. Li, H.; Pan, G. Enhanced and continued degradation of microcystins using microorganisms obtained through natural media. J. Microbiol. Methods 2014, 96, 73-80. [CrossRef] [PubMed]

80. Mankiewicz-Boczek, J.; Gagala, I.; Jurczak, T.; Jaskulska, A.; Pawelczyk, J.; Dziadek, J. Bacteria homologus to Aeromonas capable of microcystin degradation. Open Life Sci. 2015, 10, 119-129. [CrossRef]

81. Lawton, L.A.; Welgamage, A.; Manage, P.M.; Edwards, C. Novel bacterial strains for the removal of microcystins from drinking water. Water Sci. Technol. 2011, 63, 1137-1142. [CrossRef]

82. Manage, P.M.; Edwards, C.; Singh, B.K.; Lawton, L.A. Isolation and identification of novel microcystin-degrading bacteria. Appl. Environ. Microbiol. 2009, 75, 6924-6928. [CrossRef] [PubMed]

83. Alamri, S.A. Biodegradation of microcystin by a new Bacillus sp. isolated from a Saudi freshwater lake. Afr. J. Biotechnol. 2010, 9, 6552-6559.

84. Hu, L.; Zhang, F.; Liu, C.; Wang, M. Biodegradation of Microcystins by Bacillus sp. strain EMB. Energy Procedia 2012, 16, 2054-2059. [CrossRef]

85. Zhang, J.; Shi, H.; Liu, A.; Cao, Z.; Hao, J.; Gong, R. Identification of a new microcystin-degrading bacterium isolated from Lake Chaohu, China. Bull. Environ. Contam. Toxicol. 2015, 94, 661-666. [CrossRef]

86. Alamri, S.A. Biodegradation of microcystin-RR by Bacillus flexus isolated from a Saudi freshwater lake. Saudi J. Biol. Sci. 2012, 19, 435-440. [CrossRef]

87. Kansole, M.M.R.; Lin, T.F. Microcystin-LR Biodegradation by Bacillus sp.: Reaction Rates and Possible Genes Involved in the Degradation. Water 2016, 8, 508. [CrossRef]

88. Nybom, S.M.K.; Salminen, S.J.; Meriluoto, J.A.O. Removal of microcystin-LR by strains of metabolically active probiotic bacteria. FEMS Microbiol. Lett. 2007, 270, 27-33. [CrossRef] [PubMed]

89. Yang, F.; Zhou, Y.; Sun, R.; Wei, H.; Li, Y.; Yin, L.; Pu, Y. Biodegradation of microcystin-LR and-RR by a novel microcystin-degrading bacterium isolated from Lake Taihu. Biodegradation 2014, 25, 447-457. [CrossRef] [PubMed]

90. Lemes, G.A.; Kersanach, R.; Pinto, L.D.S.; Dellagostin, O.A.; Yunes, J.S.; Matthiensen, A. Biodegradation of microcystins by aquatic Burkholderia sp. from a South Brazilian coastal lagoon. Ecotoxicol. Environ. Saf. 2008, 69, 358-365. [CrossRef]

91. Shen, R.Y.; Chen, Z.H.; Dong, X.N.; Shen, H.C.; Su, P.; Mao, L.Q.; Zhang, W.Y. Biodegradation kinetics of microcystins-LR crude extract by Lysinibacillus boronitolerans strain CQ5. Ann. Microbiol. 2019, 69, 1259-1266. [CrossRef]

92. Hu, L.B.; Yang, J.D.; Zhou, W.; Yin, Y.F.; Chen, J.; Shi, Z.Q. Isolation of a Methylobacillus sp. that degrades microcystin toxins associated with cyanobacteria. New Biotechnol. 2009, 26, 205-211. [CrossRef]

93. Eleuterio, L.; Batista, J.R. Biodegradation studies and sequencing of microcystin-LR degrading bacteria isolated from a drinking water biofilter and a fresh water lake. Toxicon 2010, 55, 1434-1442. [CrossRef]

94. Phujomjai, Y.; Somdee, T. Isolation and characterization of microcystin-degrading bacterium. J. Life Sci. Technol. 2013, 1, 220-222. [CrossRef]

95. Somdee, T.; Wibuloutai, J.; Somdee, T.; Somdee, A. Biodegradation of the cyanobacterial hepatotoxin Dha(7) microcystin-LR within a biologically active sand filter. Water Sci. Technol. Water Supply 2014, 14, 672-680. [CrossRef] 
96. Phujomjai, Y.; Somdee, A.; Somdee, T. Biodegradation of microcystin Dha(7) MC-LR by a novel microcystin-degrading bacterium in an internal airlift loop bioreactor. Water Sci. Technol. 2016, 73, 267-274. [CrossRef]

97. Jiang, Y.; Shao, J.; Wu, X.; Xu, Y.; Li, R. Active and silent members in the $m l r$ gene cluster of a microcystin-degrading bacterium isolated from Lake Taihu, China. FEMS Microbiol. Lett. 2011, 322, 108-114. [CrossRef]

98. Jing, W.; Sui, G.; Liu, S. Characteristics of a Microcystin-LR Biodegrading Bacterial Isolate: Ochrobactrum sp. FDT5. Bull. Environ. Contam. Toxicol. 2014, 92, 119-122. [CrossRef] [PubMed]

99. You, D.J.; Chen, X.G.; Xiang, H.Y.; Ouyang, L.; Yang, B. Isolation, identification and characterization of a microcystin-degrading bacterium Paucibacter sp. strain CH. Huan Jing Ke Xue 2014, 35, 313-318. [PubMed]

100. Lezcano, M.A.; Moron-Lopez, J.; Agha, R.; Lopez-Heras, I.; Nozal, L.; Quesada, A.; El-Shehawy, R. Presence or Absence of $m l r$ Genes and Nutrient Concentrations Co-Determine the Microcystin Biodegradation Efficiency of a Natural Bacterial Community. Toxins 2016, 8, 318. [CrossRef] [PubMed]

101. Rapala, J.; Berg, K.A.; Lyra, C.; Niemi, R.M.; Manz, W.; Suomalainen, S.; Paulin, L.; Lahti, K. Paucibacter toxinivorans gen. nov., sp. nov., a bacterium that degrades cyclic cyanobacterial hepatotoxins microcystins and nodularin. Int. J. Syst. Evol. Microbiol. 2005, 55, 1563-1568. [CrossRef] [PubMed]

102. Takenaka, S.; Watanabe, M.F. Microcystin LR degradation by Pseudomonas aeruginosa alkaline protease. Chemosphere 1997, 34, 749-757. [CrossRef]

103. Zhang, M.L.; Yan, H.; Pan, G. Microbial degradation of microcystin-LR by Ralstonia solanacearum. Environ. Technol. 2011, 32, 1779-1787. [CrossRef]

104. Saito, T.; Okano, K.; Park, H.D.; Itayama, T.; Inamori, Y.; Neilan, B.A.; Burns, B.P.; Sugiura, N. Detection and sequencing of the microcystin LR-degrading gene, $m l r A$, from new bacteria isolated from Japanese lakes. FEMS Microbiol. Lett. 2003, 229, 271-276. [CrossRef]

105. Bourne, D.G.; Jones, G.J.; Blakeley, R.L.; Jones, A.; Negri, A.P.; Riddles, P. Enzymatic pathway for the bacterial degradation of the cyanobacterial cyclic peptide toxin microcystin LR. Appl. Environ. Microbiol. 1996, 62, 4086-4094. [CrossRef]

106. Imanishi, S.; Kato, H.; Mizuno, M.; Tsuji, K.; Harada, K. Bacterial degradation of microcystins and nodularin. Chem. Res. Toxicol. 2005, 18, 591-598. [CrossRef]

107. Harada, K.; Imanishi, S.; Kato, H.; Mizuno, M.; Ito, E.; Tsuji, K. Isolation of Adda from microcystin-LR by microbial degradation. Toxicon 2004, 44, 107-109. [CrossRef] [PubMed]

108. Kato, H.; Imanishi, S.Y.; Tsuji, K.; Harada, K. Microbial degradation of cyanobacterial cyclic peptides. Water Res. 2007, 41, 1754-1762. [CrossRef] [PubMed]

109. Valeria, A.M.; Ricardo, E.J.; Stephan, P.; Alberto, W.D. Degradation of Microcystin-RR by Sphingomonas sp. CBA4 isolated from San Roque reservoir (Cordoba-Argentina). Biodegradation 2006, 17, 447-455. [CrossRef] [PubMed]

110. Maruyama, T.; Park, H.D.; Ozawa, K.; Tanaka, Y.; Sumino, T.; Hamana, K.; Hiraishi, A.; Kato, K. Sphingosinicella microcystinivorans gen. nov., sp nov., a microcystin-degrading bacterium. Int. J. Syst. Evol. Microbiol. 2006, 56, 85-89. [CrossRef]

111. Park, H.D.; Sasaki, Y.; Maruyama, T.; Yanagisawa, E.; Hiraishi, A.; Kato, K. Degradation of the cyanobacterial hepatotoxin microcystin by a new bacterium isolated from a hypertrophic lake. Environ. Toxicol. 2001, 16, 337-343. [CrossRef]

112. Ishii, H.; Nishijima, M.; Abe, T. Characterization of degradation process of cyanobacterial hepatotoxins by a gram-negative aerobic bacterium. Water Res. 2004, 38, 2667-2676. [CrossRef]

113. Okano, K.; Shimizu, K.; Kawauchi, Y.; Maseda, H.; Utsumi, M.; Zhang, Z.; Neilan, B.A.; Sugiura, N. Characteristics of a Microcystin-Degrading Bacterium under Alkaline Environmental Conditions. J. Toxicol. 2009, 2009, 954291. [CrossRef]

114. Shimizu, K.; Maseda, H.; Okano, K.; Kurashima, T.; Kawauchi, Y.; Xue, Q.; Utsumi, M.; Zhang, Z.; Sugiura, N. Enzymatic pathway for biodegrading microcystin LR in Sphingopyxis sp. C-1. J. Biosci. Bioeng. 2012, 114, 630-634. [CrossRef]

115. Ho, L.; Hoefel, D.; Saint, C.P.; Newcombe, G. Isolation and identification of a novel microcystin-degrading bacterium from a biological sand filter. Water Res. 2007, 41, 4685-4695. [CrossRef] 
116. Ding, Q.; Liu, K.Y.; Xu, K.; Sun, R.L.; Zhang, J.; Yin, L.H.; Pu, Y.P. Further Understanding of Degradation Pathways of Microcystin-LR by an Indigenous Sphingopyxis sp. in Environmentally Relevant Pollution Concentrations. Toxins 2018, 10, 536. [CrossRef]

117. Ho, L.; Tang, T.; Monis, P.T.; Hoefel, D. Biodegradation of multiple cyanobacterial metabolites in drinking water supplies. Chemosphere 2012, 87, 1149-1154. [CrossRef] [PubMed]

118. Wang, J.; Wu, P.; Chen, J.; Yan, H. Biodegradation of Microcystin-RR by a New Isolated Sphingopyxis sp. USTB-05. Chin. J. Chem. Eng. 2010, 18, 108-112. [CrossRef]

119. Xiao, W.; Yan, W.; Jian, O.; Wei, N.; Pan, G. Microcystin-LR biodegradation by Sphingopyxis sp. USTB-05. Front. Environ. Sci. Eng. China 2011, 5, 526-532. [CrossRef]

120. Xu, H.; Wang, H.; Xu, Q.; Lv, L.; Yin, C.; Liu, X.; Du, H.; Yan, H. Pathway for Biodegrading Microcystin-YR by Sphingopyxis sp. USTB-05. PLoS ONE 2015, 10, e0124425. [CrossRef] [PubMed]

121. Yan, H.; Wang, H.; Wang, J.; Yin, C.; Ma, S.; Liu, X.; Yin, X. Cloning and expression of the first gene for biodegrading microcystin LR by Sphingopyxis sp. USTB-05. J. Environ. Sci. 2012, 24, 1816-1822. [CrossRef]

122. Qin, L.; Zhang, X.X.; Chen, X.G.; Wang, K.; Shen, Y.T.; Li, D. Isolation of a Novel Microcystin-Degrading Bacterium and the Evolutionary Origin of $m l r$ Gene Cluster. Toxins 2019, 11, 269. [CrossRef]

123. Yang, F.; Huang, F.; Feng, H.; Wei, J.; Massey, I.Y.; Liang, G.; Zhang, F.; Yin, L.; Kacew, S.; Zhang, X.; et al. A complete route for biodegradation of potentially carcinogenic cyanotoxin microcystin-LR in a novel indigenous bacterium. Water Res. 2020, 174, 115638. [CrossRef]

124. Chen, J.; Hu, L.B.; Zhou, W.; Yan, S.H.; Yang, J.D.; Xue, Y.F.; Shi, Z.Q. Degradation of microcystin-LR and RR by a Stenotrophomonas sp. strain EMS isolated from Lake Taihu, China. Int. J. Mol. Sci. 2010, 11, 896-911. [CrossRef]

125. Yang, F.; Zhou, Y.; Yin, L.; Zhu, G.; Liang, G.; Pu, Y. Microcystin-degrading activity of an indigenous bacterial strain Stenotrophomonas acidaminiphila MC-LTH2 isolated from Lake Taihu. PLoS ONE 2014, 9, e86216. [CrossRef]

126. Manheim, D.; Cheung, Y.M.; Jiang, S. The Effect of Organic Carbon Addition on the Community Structure and Kinetics of Microcystin-Degrading Bacterial Consortia. Water 2018, 10, 1523. [CrossRef]

127. Kumar, P.; Hegde, K.; Brar, S.K.; Cledon, M.; Kermanshahi-Pour, A.; Roy-Lachapelle, A.; Galvez-Cloutier, R. Biodegradation of microcystin-LR using acclimatized bacteria isolated from different units of the drinking water treatment plant. Environ. Pollut. 2018, 242, 407-416. [CrossRef] [PubMed]

128. Yang, F.; Guo, J.; Huang, F.; Massey, I.Y.; Huang, R.; Li, Y.; Wen, C.; Ding, P.; Zeng, W.; Liang, G. Removal of Microcystin-LR by a Novel Native Effective Bacterial Community Designated as YFMCD4 Isolated from Lake Taihu. Toxins 2018, 10, 363. [CrossRef]

129. Yang, F.; Massey, I.Y.; Guo, J.; Yang, S.; Pu, Y.; Zeng, W.; Tan, H. Microcystin-LR degradation utilizing a novel effective indigenous bacterial community YFMCD1 from Lake Taihu. J. Toxicol. Environ. Health A 2018, 1-10. [CrossRef]

130. Tsao, S.; Wei, D.J.; Chang, Y.T.; Lee, J.F. Aerobic biodegradation biodegradation of microcystin-LR by an indigenous bacterial mixed culture isolated in Taiwan. Int. Biodeterior. Biodegrad. 2017, 124, 101-108. [CrossRef]

131. Briand, E.; Humbert, J.F.; Tambosco, K.; Bormans, M.; Gerwick, W.H. Role of bacteria in the production and degradation of Microcystis cyanopeptides. Microbiologyopen 2016, 5, 469-478. [CrossRef]

132. Mou, X.; Lu, X.; Jacob, J.; Sun, S.; Heath, R. Metagenomic Identification of Bacterioplankton Taxa and Pathways Involved in Microcystin Degradation in Lake Erie. PLoS ONE 2013, 8. [CrossRef] [PubMed]

133. Ramani, A.; Rein, K.; Shetty, K.G.; Jayachandran, K. Microbial degradation of microcystin in Florida's freshwaters. Biodegradation 2012, 23, 35-45. [CrossRef]

134. Christoffersen, K.; Lyck, S.; Winding, A. Microbial activity and bacterial community structure during degradation of microcystins. Aquat. Microb. Ecol. 2002, 27, 125-136. [CrossRef] 
135. Bourne, D.G.; Riddles, P.; Jones, G.J.; Smith, W.; Blakeley, R.L. Characterisation of a gene cluster involved in bacterial degradation of the cyanobacterial toxin microcystin LR. Environ. Toxicol. 2001, 16, 523-534. [CrossRef]

136. Dziga, D.; Wasylewski, M.; Szetela, A.; Bocheńska, O.; Wladyka, B. Verification of the Role of MlrC in Microcystin Biodegradation by Studies Using a Heterologously Expressed figureEnzyme. Chem. Res. Toxicol. 2012, 25, 1192-1194. [CrossRef]

(C) 2020 by the authors. Licensee MDPI, Basel, Switzerland. This article is an open access article distributed under the terms and conditions of the Creative Commons Attribution (CC BY) license (http://creativecommons.org/licenses/by/4.0/). 